\title{
MOCK MODULAR PERIOD FUNCTIONS AND $L$-FUNCTIONS OF CUSP FORMS IN HIGHER LEVEL CASES
}

\author{
SOYOUNG CHOI AND CHANG HEON KIM
}

(Communicated by Ken Ono)

\begin{abstract}
Generalizing the results of Bringmann, Guerzhoy, Kent and Ono, we investigate mock modular period polynomials associated to harmonic Maass forms for $\Gamma_{0}^{+}(p)$. In particular, using period relations generated from the period polynomials, we derive congruence relations involving the critical values of modular $L$-functions and show that these congruence relations are indeed equalities by using integral representations of nonholomorphic parts of harmonic Maass forms.
\end{abstract}

\section{Introduction AND STATEMENT OF RESUlts}

Let $p$ be one or a prime and $\Gamma_{0}^{+}(p)$ be the group generated by the Hecke group $\Gamma_{0}(p)$ and the Fricke involution $W_{p}=\left(\begin{array}{cc}0 & -1 / \sqrt{p} \\ \sqrt{p} & 0\end{array}\right)$. Let $M_{k}^{!}\left(\Gamma_{0}^{+}(p)\right)$ be the vector space of weakly holomorphic modular forms (that is, meromorphic with poles only at the cusp infinity) of weight $k$ for $\Gamma_{0}^{+}(p)$ and $S_{k}^{!}\left(\Gamma_{0}^{+}(p)\right)$ be its subspace consisting of forms with zero constant terms. For $F(z) \in M_{k}^{!}\left(\Gamma_{0}^{+}(p)\right)$ with Fourier expansion

$$
F(z)=\sum_{n \gg-\infty} a_{F}(n) q^{n} \quad\left(q=e^{2 \pi i z}\right)
$$

we define

$$
\mathcal{E}_{F}(z):=\sum_{n \gg-\infty, n \neq 0} a_{F}(n) n^{1-k} q^{n} .
$$

The period function for $F$ is then defined by

$$
r^{+}(F ; z)=r^{+}\left(F, W_{p} ; z\right):=c_{k}\left(\mathcal{E}_{F}-\left.\mathcal{E}_{F}\right|_{2-k} W_{p}\right)(z),
$$

where $c_{k}:=-\frac{\Gamma(k-1)}{(2 \pi i)^{k-1}}$. We note that for $F \in S_{k}^{!}\left(\Gamma_{0}^{+}(p)\right)$ the function $r^{+}(F ; z)$ is a polynomial in $z$ of degree at most $k-2$ with coefficients in $\mathbb{C}$. Indeed this fact can

Received by the editors September 5, 2012 and, in revised form, September 19, 2012 and October 20, 2012.

2010 Mathematics Subject Classification. Primary 11F11, 11F67; Secondary 11F37.

Key words and phrases. Weakly holomorphic modular forms, mock modular forms, period polynomials.

The first author was supported by the Dongguk University Research fund of 2013 and by the Basic Science Research Program through the National Research Foundation of Korea (NRF) funded by the Ministry of Education, Science and Technology (NRF-2012R1A1A3011711).

The second author was supported by the Basic Science Research Program through the National Research Foundation of Korea (NRF) funded by the Ministry of Education, Science and Technology (NRF-2013R1A1A2A10004632). 
be shown by Bol's identity [1]. Let $F, G \in M_{k}^{!}\left(\Gamma_{0}^{+}(p)\right)$ have Fourier expansions as follows:

$$
F(z)=\sum_{n \gg-\infty} a_{F}(n) q^{n} \text { and } G(z)=\sum_{n \gg-\infty} a_{G}(n) q^{n} .
$$

We define a pairing $\{F, G\}$ by

$$
\{F, G\}:=\sum_{n \in \mathbb{Z}, n \neq 0} \frac{a_{F}(-n) a_{G}(n)}{n^{k-1}} .
$$

This antisymmetric, bilinear and Hecke equivariant pairing is a generalization of pairings in [6, p.2024] and [8, p.3053], where it was used to derive congruences for Hecke eigenvalues. Specifically, for any prime $r(\neq p)$,

$$
\left\{T_{r} F, G\right\}=\left\{F, T_{r} G\right\},
$$

where $T_{r} F=\sum\left(a_{F}(r n)+r^{k-1} a_{F}(n / r)\right) q^{n}$.

Recently Brigmann, Guerzhoy, Kent and Ono [2] studied questions concerning periods and harmonic Maass forms for the modular group $S L_{2}(\mathbb{Z})$ and showed that the pairing (11) is related to period polynomials $r^{+}(F ; z)$ that extend the classical period maps. Furthermore they derived period relations between period polynomials for forms associated to harmonic weak Maass forms and used mock modular forms to compute generating functions for the critical values of modular $L$-functions. In this paper we generalize their results to higher level groups $\Gamma_{0}^{+}(p)$ with $p \in\{2,3\}$. Our first result is an extension of [2, Theorem 1.6], where we find equivalent conditions for the period polynomial $r^{+}(F ; z)$ to be a multiple of $(\sqrt{p} z)^{k-2}-1$.

Theorem 1.1. Let $p=2$ or 3 and $F \in S_{k}^{!}\left(\Gamma_{0}^{+}(p)\right)$ with Fourier expansion $F(z)=$ $\sum_{n \in \mathbb{Z}} a_{F}(n) q^{n}$. Denote by $D$ the differential operator $D=q \frac{d}{d q}=\frac{1}{2 \pi i} \frac{d}{d z}$. Then the following conditions are equivalent:

(1) $F \in D^{k-1}\left(M_{2-k}^{!}\left(\Gamma_{0}^{+}(p)\right)\right)$,

(2) $r^{+}(F ; z) \equiv 0\left(\bmod (\sqrt{p} z)^{k-2}-1\right)$,

(3) $\{F, g\}=0$, for every $g \in S_{k}^{!}\left(\Gamma_{0}^{+}(p)\right)$.

Remark 1.2. The reason for considering the cases $p=2$ or 3 in Theorem 1.1 is as follows. In order to prove the theorem we need Lemma 2.1 where we use the fact that $\Gamma_{0}^{+}(p) /\{ \pm 1\}$ is generated by two elements $T$ and $W_{p}$, for which the condition $p=2$ or 3 is required (see [12]).

Let $H_{2-k}\left(\Gamma_{0}(p)\right)$ be the space of harmonic weak Maass forms on $\Gamma_{0}(p)$ and $H_{2-k}^{*}\left(\Gamma_{0}(p)\right)$ be the subspace of $H_{2-k}\left(\Gamma_{0}(p)\right)$ consisting of elements $\mathcal{F}$ with the property that for each cusp $s$ there is a polynomial $P_{\mathcal{F}, s}(z)=\sum_{n \leq 0} a_{\mathcal{F}}^{+}(n) q_{h_{s}}^{n} \in$ $\mathbb{C}\left[q_{h_{s}}^{-1}\right]$ such that $\left.\mathcal{F}(z)\right|_{k} \sigma_{s}^{-1}-P_{\mathcal{F}, s}(z)=O\left(e^{-\epsilon y}\right)$ as $y=\operatorname{Im}(z) \rightarrow \infty$ for some $\epsilon>0$. Here $h_{s}$ denotes the width of the cusp $s$ and $\sigma_{s}$ is an element of $S L_{2}(\mathbb{Z})$ with $\sigma_{s}(s)=\infty$. Every harmonic weak Maass form $\mathcal{F} \in H_{2-k}\left(\Gamma_{0}(p)\right)$ has a unique decomposition [4, 10]

$$
\mathcal{F}(z)=\mathcal{F}^{-}(z)+\mathcal{F}^{+}(z),
$$

where $\mathcal{F}^{-}$(respectively $\mathcal{F}^{+}$) is nonholomorphic (respectively holomorphic) on the complex upper half-plane $\mathbb{H}$. The holomorphic part $\mathcal{F}^{+}$has Fourier expansion

$$
\mathcal{F}^{+}(z)=\sum_{n \gg-\infty} a_{\mathcal{F}}^{+}(n) q^{n} \quad\left(q^{n}:=e^{2 \pi i z}\right) .
$$


Then we call $\mathcal{F}^{+}$a mock modular form if $\mathcal{F}^{-} \neq 0$. We define

$$
H_{2-k}\left(\Gamma_{0}^{+}(p)\right):=\left\{\mathcal{F} \in H_{2-k}\left(\Gamma_{0}(p)\right)|\mathcal{F}|_{2-k} W_{p}=\mathcal{F}\right\}
$$

and

$$
H_{2-k}^{*}\left(\Gamma_{0}^{+}(p)\right):=\left\{\mathcal{F} \in H_{2-k}^{*}\left(\Gamma_{0}(p)\right)|\mathcal{F}|_{2-k} W_{p}=\mathcal{F}\right\} .
$$

For each $\mathcal{F} \in H_{2-k}^{*}\left(\Gamma_{0}^{+}(p)\right)$ we define the $W_{p}$-mock modular period function for $\mathcal{F}^{+}$ by

$$
\mathbb{P}\left(\mathcal{F}^{+}, W_{p} ; z\right):=\frac{(4 \pi)^{k-1}}{\Gamma(k-1)}\left(\mathcal{F}^{+}-\left.\mathcal{F}^{+}\right|_{2-k} W_{p}\right)(z) .
$$

Let $L_{k}$ be the Maass lowering operator $L_{k}$ defined by

$$
L_{k}=-2 i y^{2} \frac{\partial}{\partial \bar{\tau}}=-i y^{2}\left(\frac{\partial}{\partial x}+i \frac{\partial}{\partial y}\right) .
$$

Then for $k \geq 2$ the differential operator $\xi_{2-k}:=y^{k} \overline{L_{2-k}}$ defines antilinear maps

$$
\xi_{2-k}: H_{2-k}\left(\Gamma_{0}^{+}(p)\right) \rightarrow M_{k}^{!}\left(\Gamma_{0}^{+}(p)\right) \quad \text { and } \quad \xi_{2-k}: H_{2-k}^{*}\left(\Gamma_{0}^{+}(p)\right) \rightarrow S_{k}\left(\Gamma_{0}^{+}(p)\right)
$$

(see Proposition [3.3). As extensions of [2, Theorems 1.4 and 1.1] we have the following theorems. The next theorem provides period relations between period polynomials for forms associated to given mock modular forms.

Theorem 1.3. Let $p=2$ or 3 . If $\mathcal{F} \in H_{2-k}\left(\Gamma_{0}^{+}(p)\right)$, then we have that

$$
r^{+}\left(\xi_{2-k}(\mathcal{F}) ; z\right) \equiv \frac{-(4 \pi)^{k-1}}{\Gamma(k-1)} \overline{r^{+}\left(D^{k-1}(\mathcal{F}) ; \bar{z}\right)} \quad\left(\bmod (\sqrt{p} z)^{k-2}-1\right),
$$

where equivalence modulo $(\sqrt{p} z)^{k-2}-1$ means that the difference of the two functions is a constant multiple of $(\sqrt{p} z)^{k-2}-1$. Moreover, there is a function $\widehat{\mathcal{F}} \in$ $H_{2-k}\left(\Gamma_{0}^{+}(p)\right)$ for which

$$
\xi_{2-k}(\mathcal{F})=\xi_{2-k}(\widehat{\mathcal{F}}) \text { and } r^{+}\left(\xi_{2-k}(\mathcal{F}) ; z\right)=\frac{-(4 \pi)^{k-1}}{\Gamma(k-1)} \overline{r^{+}\left(D^{k-1}(\widehat{\mathcal{F}}) ; \bar{z}\right)} .
$$

Remark 1.4. The reason for considering the cases $p=2$ or 3 in Theorem 1.3 is as follows. In the course of proving the theorem we are in need of Propositions $3.1,3.2$ and 3.4, where a complex number $\zeta \in \mathbb{H}$ satisfying the equation $\zeta^{2}-\sqrt{p} \zeta+1=0$ is required. The condition $p \leq 3$ is needed for the existence of such a complex number $\zeta$.

As our last result we compute generating functions for the critical values of modular $L$-functions using their corresponding mock modular forms.

Theorem 1.5. For $\mathcal{F} \in H_{2-k}^{*}\left(\Gamma_{0}^{+}(p)\right)$ and $f=\xi_{2-k}(\mathcal{F})$ we have that

$$
\overline{\mathbb{P}\left(\mathcal{F}^{+}, W_{p} ; \bar{z}\right)}=c_{k}^{-1} r^{+}(f ; z)=\sum_{n=0}^{k-2} \frac{L(f, n+1)}{(k-2-n) !}(2 \pi i z)^{k-2-n} .
$$

This paper is organized as follows. The proofs of Theorem 1.1 and Theorem 1.3 are given in Section 2 and Section 3, respectively. In Section 4 we derive a congruence relation regarding the two polynomials in Theorem 1.5 and then we will prove that this congruence relation is indeed an equality by using an integral representation of a nonholomorphic part of a harmonic Maass form. We note that the proof of Theorem 1.5] also provides a simple proof of [2, Theorem 1.1]. 


\section{Proof of Theorem 1.1}

In what follows we denote $\left(\begin{array}{ll}1 & 1 \\ 0 & 1\end{array}\right)$ by $T$. We need two lemmas to prove Theorem 1.1

Lemma 2.1. Let $p=2$ or 3 . We have that $r^{+}(F ; z)=\alpha\left((\sqrt{p} z)^{k-2}-1\right)$ for some $\alpha \in \mathbb{C}$ if and only if $\mathcal{E}_{F}+\frac{\alpha}{c_{k}} \in M_{2-k}^{!}\left(\Gamma_{0}^{+}(p)\right)$.

Proof. Suppose that $r^{+}(F ; z)=\alpha\left((\sqrt{p} z)^{k-2}-1\right)$ for some $\alpha \in \mathbb{C}$. Then we have that

$$
\mathcal{E}_{F}+\frac{\alpha}{c_{k}}=\left.\left(\mathcal{E}_{F}+\frac{\alpha}{c_{k}}\right)\right|_{2-k} W_{p} .
$$

By definition of $\mathcal{E}_{F}$, we see that $\mathcal{E}_{F}+\frac{\alpha}{c_{k}}=\left.\left(\mathcal{E}_{F}+\frac{\alpha}{c_{k}}\right)\right|_{2-k} T$. Moreover, since $\Gamma_{0}^{+}(p)$ is generated by $\pm T$ and $\pm W_{p}$, we obtain that $\mathcal{E}_{F}+\frac{\alpha}{c_{k}} \in M_{2-k}^{!}\left(\Gamma_{0}^{+}(p)\right)$. Now suppose that $\mathcal{E}_{F}+\frac{\alpha}{c_{k}} \in M_{2-k}^{!}\left(\Gamma_{0}^{+}(p)\right)$ for some $\alpha \in \mathbb{C}$. This implies that

$$
\begin{aligned}
\frac{1}{c_{k}} r^{+}(F ; z) & =\left(\mathcal{E}_{F}-\left.\mathcal{E}_{F}\right|_{2-k} W_{p}\right)(z) \\
& =\left(\left.\frac{\alpha}{c_{k}}\right|_{2-k} W_{p}\right)(z)-\frac{\alpha}{c_{k}} \\
& =\frac{\alpha}{c_{k}}\left((\sqrt{p} z)^{k-2}-1\right) .
\end{aligned}
$$

We introduce the regularized inner product $(f, h)^{r e g}$ for $f \in M_{k}^{!}\left(\Gamma_{0}(p)\right)$ and $h \in M_{k}\left(\Gamma_{0}(p)\right)$. It is defined to be the constant term in the Laurent expansion at $s=0$ of the meromorphic continuation in $s$ of the function

$$
\lim _{\epsilon \rightarrow 0^{+}} \int_{F_{p}(\epsilon)} f(\tau) \overline{h(\tau)} y^{k-s} \frac{d x d y}{y^{2}} .
$$

As in [5, p.5], if $f \in M_{k}^{!}\left(\Gamma_{0}(p)\right)$ has a vanishing constant term at every cusp of $\Gamma_{0}(p)$, then

$$
(f, h)^{r e g}=\lim _{\epsilon \rightarrow 0^{+}} \int_{F_{p}(\epsilon)} f(\tau) \overline{h(\tau)} y^{k} \frac{d x d y}{y^{2}}
$$

where

$$
\mathcal{F}(\epsilon)=\left\{\tau \in \mathbb{H}|| x\left|\leq \frac{1}{2},\right| \tau \mid \geq 1, y \leq \frac{1}{\epsilon}\right\}
$$

and

$$
\mathcal{F}_{p}(\epsilon)=\bigcup_{\gamma \in \Gamma_{0}(p) \backslash S L_{2}(\mathbb{Z})} \gamma \mathcal{F}(\epsilon) .
$$

Let $C_{p}$ denote the set of all cusps for $\Gamma_{0}(p)$. For each cusp $s \in C_{p}$, take $\sigma_{s} \in$ $S L_{2}(\mathbb{Z})$ so that $\sigma_{s}(s)=\infty$. Then, at every cusp $s \in C_{p}, f \in M_{k}^{!}\left(\Gamma_{0}(p)\right)$ and $g \in H_{2-k}\left(\Gamma_{0}(p)\right)$ have the Fourier expansions of the form [4, 10 .

$$
\begin{aligned}
\left(\left.f\right|_{k} \sigma_{s}^{-1}\right)(\tau) & =\sum_{n \gg-\infty} c_{f}(s, n) q_{h_{s}}^{n}, \\
\left(\left.g\right|_{2-k} \sigma_{s}^{-1}\right)(\tau) & =\sum_{n \gg-\infty} c_{g}^{+}(s, n) q_{h_{s}}^{n}+\sum_{n \ll \infty} c_{g}^{-}(s, n) \Gamma\left(k-1,-4 \pi n y / h_{s}\right) q_{h_{s}}^{n},
\end{aligned}
$$

where $q_{h_{s}}=e^{2 \pi i \tau / h_{s}}$ and $\Gamma(s, w)=\int_{w}^{\infty} e^{-t} t^{s} \frac{d t}{t}$ is the incomplete Gamma function. 
Lemma 2.2. Let $f \in M_{k}^{!}\left(\Gamma_{0}(p)\right)$ and $g \in H_{2-k}\left(\Gamma_{0}(p)\right)$. Suppose that $f$ has vanishing constant terms at all cusps of $\Gamma_{0}(p)$. Then with the notation as above,

$$
\left(f, \xi_{2-k}(g)\right)^{r e g}=\sum_{s \in C_{p}} h_{s}\left(\sum_{m+n=0} c_{f}(s, m) c_{g}^{+}(s, n)\right) .
$$

Proof. See [5, Theorem 3.1].

Proof of Theorem 1.1. (1) $\Leftrightarrow(2)$ : let $F \in D^{k-1}\left(M_{2-k}^{!}\left(\Gamma_{0}^{+}(p)\right)\right)$ with $F=D^{k-1} G$ for some $G \in M_{2-k}^{!}\left(\Gamma_{0}^{+}(p)\right)$. Noticing that

$$
\mathcal{E}_{F}(z)=\sum_{n \gg-\infty, n \neq 0} a_{F}(n) n^{1-k} q^{n}=G(z)-a_{G}(0)
$$

we have

$$
\begin{aligned}
r^{+}(F ; z) & =c_{k}\left(G(z)-a_{G}(0)-\left.\left(G(z)-a_{G}(0)\right)\right|_{2-k} W_{p}\right)(z) \\
& =c_{k} a_{G}(0)\left((\sqrt{p} z)^{k-2}-1\right) .
\end{aligned}
$$

We now suppose that $r^{+}(F ; z)=\alpha\left((\sqrt{p} z)^{k-2}-1\right)$ for some $\alpha \in \mathbb{C}$. By Lemma 2.1 we have

$$
\mathcal{E}_{F}+\frac{\alpha}{c_{k}} \in M_{2-k}^{!}\left(\Gamma_{0}^{+}(p)\right) \text { and } D^{k-1}\left(\mathcal{E}_{F}+\frac{\alpha}{c_{k}}\right)=D^{k-1} \mathcal{E}_{F}=F,
$$

which implies $F \in D^{k-1}\left(M_{2-k}^{!}\left(\Gamma_{0}^{+}(p)\right)\right)$. Thus (1) and (2) are equivalent.

$(1) \Leftrightarrow(3)$ : Let $F \in D^{k-1}\left(M_{2-k}^{!}\left(\Gamma_{0}^{+}(p)\right)\right)$ with $F=D^{k-1} G$ for some $G \in$ $M_{2-k}^{!}\left(\Gamma_{0}^{+}(p)\right)$ with Fourier expansion $G(z)=\sum_{n \in \mathbb{Z}} a_{G}(n) q^{n}$. Since $F=D^{k-1} G$ we have that $a_{F}(n)=n^{k-1} a_{G}(n)$ for each $n \in \mathbb{Z}$. Hence we obtain that

$$
\{F, g\}=\sum_{\substack{n \in \mathbb{Z} \\ n \neq 0}} \frac{a_{F}(-n) a_{g}(n)}{n^{k-1}}=-\sum_{\substack{n \in \mathbb{Z} \\ n \neq 0}} a_{G}(-n) a_{g}(n)=0,
$$

for all $g \in S_{k}^{!}\left(\Gamma_{0}^{+}(p)\right)$. The last equality comes from a residue theorem for $G g \in$ $M_{2}^{!}\left(\Gamma_{0}^{+}(p)\right)$. Now assume that $\{F, g\}=0$ for all $g \in S_{k}^{!}\left(\Gamma_{0}^{+}(p)\right)$. We have only to show that $F \in D^{k-1}\left(M_{2-k}^{!}\left(\Gamma_{0}^{+}(p)\right)\right)$. Let $t=\operatorname{dim} S_{k}\left(\Gamma_{0}^{+}(p)\right)$. Then for each integer $m$ such that $-m \leq t$, there exists a unique weakly holomorphic modular form $f_{k, m} \in M_{k}^{!}\left(\Gamma_{0}^{+}(p)\right)$ with $q$-expansion of the form

$$
f_{k, m}=q^{-m}+O\left(q^{t+1}\right) .
$$

Moreover these functions form a basis for $M_{k}^{!}\left(\Gamma_{0}^{+}(p)\right)$ (see [7, Theorem 2.7]). Similarly for each integer $m$ such that $-m \leq-t-1$, there exists a unique weakly holomorphic modular form $f_{2-k, m} \in M_{k}^{!}\left(\Gamma_{0}^{+}(p)\right)$ with $q$-expansion of the form

$$
f_{2-k, m}=q^{-m}+O\left(q^{-t}\right) .
$$

Moreover these functions also form a basis for $M_{2-k}^{!}\left(\Gamma_{0}^{+}(p)\right)$ (see [7, Theorem 2.7]). Let

$$
F=\sum_{-t \leq n \leq t_{0}} a_{F}(-n) f_{k, n}
$$

for some $t_{0} \in \mathbb{Z}$. If $t=0$, then

$$
F-\sum_{t+1 \leq m \leq t_{0}} \frac{a_{F}(-m)}{(-m)^{k-1}} D^{k-1}\left(f_{2-k, m}\right)=O\left(q^{t+1}\right) .
$$


Thus the left hand side of (2) should be zero, and $F$ belongs to the space $D^{k-1}\left(M_{2-k}^{!}\left(\Gamma_{0}^{+}(p)\right)\right)$. Now we suppose that $t \geq 1$. First we consider the case $t+1 \leq t_{0}$. Letting $g_{m}=\frac{1}{(-m)^{k-1}} D^{k-1} f_{2-k, m}$ for each $m \geq t+1$ we have that

$$
F-\sum_{t+1 \leq m \leq t_{0}} a_{F}(-m) g_{m}=O\left(q^{-t}\right) \text {. }
$$

Moreover since for each $g \in S_{k}^{!}\left(\Gamma_{0}^{+}(p)\right)$,

$$
\begin{aligned}
\left\{g_{m}, g\right\} & =\sum_{n \in \mathbb{Z}, n \neq 0}(-n)^{k-1} \frac{a_{2-k}(m,-n) a_{g}(n)}{n^{k-1}(-m)^{k-1}}=\frac{1}{m^{k-1}} \sum_{i+j=0} a_{2-k}(m, i) a_{g}(j) \\
& =\frac{1}{m^{k-1}(p+1)}\left(f_{2-k, m}, \xi_{2-k}(g)\right)^{r e g} \\
& =\frac{1}{m^{k-1}(p+1)}\left(f_{2-k, m}, 0\right)^{r e g}=0,
\end{aligned}
$$

we have that

$$
\left\{F-\sum_{t+1 \leq m \leq t_{0}} a_{F}(-m) g_{m}, g\right\}=\{F, g\}=0 .
$$

Here the equality (3) comes from Lemma 2.2. Take $G_{n}=f_{k, n}$ for each $-t \leq n \leq t$ with $n \neq 0$. Then we have that

$$
0=\left\{F, G_{n}\right\}=\left\{F-\sum_{t+1 \leq m \leq t_{0}} a_{F}(-m) g_{m}, G_{n}\right\}=\frac{a_{F}(n)}{(-n)^{k-1}} .
$$

This means that $F(z)-\sum_{t+1 \leq m \leq t_{0}} a_{F}(-m) g_{m}(z)=O\left(q^{t+1}\right)$, and hence we obtain $F=\sum_{t+1 \leq m \leq t_{0}} a_{F}(-m) g_{m}=\sum_{t+1 \leq m \leq t_{0}} \frac{a_{F}(-m)}{(-m)^{k-1}} D^{k-1} f_{2-k, m} \in D^{k-1}\left(M_{2-k}^{!}\left(\Gamma_{0}^{+}(p)\right)\right)$.

If $t_{0} \leq t$, then $F(z)=O\left(q^{-t}\right)$, and hence we obtain $0=\left\{F, f_{k, n}\right\}=\frac{a_{F}(n)}{(-n)^{k-1}}$ for each $-t \leq n \leq t$ with $n \neq 0$, which implies $F=0$. Consequently the assertion holds.

\section{Proof of Theorem 1.3}

For $G \in M_{k}^{!}\left(\Gamma_{0}^{+}(p)\right)$, we define the involution $G^{c}$ as

$$
G^{c}(z):=\overline{G(-\bar{z})} \text {. }
$$

Then we can easily see that $G^{c} \in M_{k}^{!}\left(\Gamma_{0}^{+}(p)\right)$,

$$
\mathcal{E}_{G^{c}}(z)=\overline{\mathcal{E}_{G^{c}}(-\bar{z})} \text { and } r^{+}\left(G^{c} ; z\right)=-\overline{r^{+}(G,-\bar{z})} .
$$

When $p$ is 2 (respectively, 3 ), for $G \in S_{k}^{!}\left(\Gamma_{0}^{+}(p)\right)$ we define

and

$$
\begin{gathered}
\zeta=e^{\frac{\pi i}{4}} \quad\left(\text { respectively, } e^{\frac{\pi i}{6}}\right), \\
H_{G}^{+}(z):=\int_{-\frac{1}{\sqrt{p} \zeta}}^{\zeta / \sqrt{p}} G(\tau)(\tau-z)^{k-2} d \tau
\end{gathered}
$$

Then we have that

$$
\mathcal{E}_{G}^{+\zeta}(z):=\int_{z}^{\zeta / \sqrt{p}} G(\tau)(\tau-z)^{k-2} d \tau
$$

$$
D^{k-1}\left(\mathcal{E}_{G}^{+\zeta}(z)\right)=c_{k} G(z),
$$


which means that $\mathcal{E}_{G}^{+\zeta}(z)=c_{k} \mathcal{E}_{G}^{+}(z)+q_{G}^{+}$for some polynomial $q_{G}^{+}$of degree at most $k-2$. In the following proposition we investigate some properties of the functions $H_{G}^{+}, q_{G}^{+}$and $r^{+}(G ; z)$ regarding the actions of the matrices $W_{p}$ and $T$.

Proposition 3.1. Let $p=2$ or 3 and $G \in S_{k}^{!}\left(\Gamma_{0}^{+}(p)\right)$. Then we have the following:

(1) $H_{G}^{+}(z)=\left(\left.\mathcal{E}_{G}^{+\zeta}\right|_{2-k}\left(1-W_{p}\right)\right)(z)=\left(\left.\mathcal{E}_{G}^{+\zeta}\right|_{2-k}(1-T)\right)(z)$.

(2) $\left.H_{G}^{+}\right|_{2-k}\left(1+W_{p}\right)=0$.

(3) $H_{G}^{+}(z)=\left(\left.q_{G}^{+}\right|_{2-k}(1-T)\right)(z)=r^{+}(G ; z)+\left(\left.q_{G}^{+}\right|_{2-k}\left(1-W_{p}\right)\right)(z)$.

(4) $r^{+}(G ; z)=\left(\left.q_{G}^{+}\right|_{2-k}\left(W_{p}-T\right)\right)(z)$.

Proof. For (1) we compute that

$$
\begin{aligned}
H_{G}^{+}(z) & =\int_{z}^{\zeta / \sqrt{p}} G(\tau)(\tau-z)^{k-2} d \tau-\int_{z}^{-1 / \sqrt{p} \zeta} G(\tau)(\tau-z)^{k-2} d \tau \\
& =\int_{z}^{\zeta / \sqrt{p}} G(\tau)(\tau-z)^{k-2} d \tau-\int_{-\frac{1}{p z}}^{\zeta / \sqrt{p}} G(\tau)(p \tau z+1)^{k-2} \sqrt{p}^{2-k} d \tau \\
& =\int_{z}^{\zeta / \sqrt{p}} G(\tau)(\tau-z)^{k-2} d \tau-(\sqrt{p} z)^{k-2} \int_{-\frac{1}{p z}}^{\zeta / \sqrt{p}} G(\tau)\left(\tau+\frac{1}{p z}\right)^{k-2} d \tau \\
& =\left(\left.\mathcal{E}_{G}^{+\zeta}\right|_{2-k}\left(1-W_{p}\right)\right)(z) .
\end{aligned}
$$

Moreover we have that

$$
\begin{aligned}
\left(\left.\mathcal{E}_{G}^{+\zeta}\right|_{2-k} T\right)(z) & =\int_{z+1}^{\zeta / \sqrt{p}} G(\tau)(z+1-\tau)^{k-2} d \tau=\int_{z}^{-\frac{1}{\sqrt{p} \zeta}} G(\tau)(z-\tau)^{k-2} d \tau \\
& =\left(\left.\mathcal{E}_{G}^{+\zeta}\right|_{2-k} W_{p}\right)(z) .
\end{aligned}
$$

Hence we obtain the first assertion. (2) follows from (1).

To prove (3) we use the fact that $\mathcal{E}_{G}^{+\zeta}(z)=c_{k} \mathcal{E}_{G}^{+}(z)+q_{G}^{+}(z)$ and (1). We then have that

$$
\begin{aligned}
H_{G}^{+}(z) & =\left(\left.\mathcal{E}_{G}^{+\zeta}\right|_{2-k}(1-T)\right)(z) \\
& =\left(\left.c_{k} \mathcal{E}_{G}^{+}\right|_{2-k}(1-T)\right)(z)+\left.q_{G}^{+}\right|_{2-k}(1-T)(z) \\
& =\left.q_{G}^{+}\right|_{2-k}(1-T)(z)
\end{aligned}
$$

and

$$
\begin{aligned}
H_{G}^{+}(z) & =\left(\left.\mathcal{E}_{G}^{+\zeta}\right|_{2-k}\left(1-W_{p}\right)\right)(z) \\
& =\left(\left.c_{k} \mathcal{E}_{G}^{+}\right|_{2-k}\left(1-W_{p}\right)\right)(z)+\left.q_{G}^{+}\right|_{2-k}\left(1-W_{p}\right)(z) \\
& =r^{+}(G ; z)+\left.q_{G}^{+}\right|_{2-k}\left(1-W_{p}\right)(z) .
\end{aligned}
$$

(4) follows from (3).

Let $p=2$ or 3 and $G \in S_{k}^{!}\left(\Gamma_{0}^{+}(p)\right)$. We define

$$
\Phi_{G}^{+}(z):=\int_{-\bar{z}}^{\zeta / \sqrt{p}} G(\tau)(\tau+z)^{k-2} d \tau .
$$

Proposition 3.2. Let $p=2$ or 3 and $G \in S_{k}^{!}\left(\Gamma_{0}^{+}(p)\right)$. Then we have that

(1) $\left(\left.\Phi_{G}^{+}\right|_{2-k} T^{-1}\right)(z)=\left(\left.\Phi_{G}^{+}\right|_{2-k} W_{p}\right)(z)=\int_{-\bar{z}}^{-\frac{1}{\sqrt{p} \zeta}} G(\tau)(\tau+z)^{k-2} d \tau$.

(2) $H_{G}^{+}(-z)=\left(\left.\Phi_{G}^{+}\right|_{2-k}\left(1-T^{-1}\right)\right)(z)=\left(\left.\Phi_{G}^{+}\right|_{2-k}\left(1-W_{p}\right)\right)(z)$. 
Proof. For (1) we find that

$$
\begin{aligned}
\left(\left.\Phi_{G}^{+}\right|_{2-k} T^{-1}\right)(z) & =\int_{-\overline{(z-1)}}^{\zeta / \sqrt{p}} G(\tau)(\tau+z-1)^{k-2} d \tau=\int_{-\bar{z}}^{-\frac{1}{\zeta \sqrt{p}}} G(\tau)(\tau+z)^{k-2} d \tau \\
& =\sqrt{p}^{k-2} \int_{-\bar{z}}^{-\frac{1}{\zeta \sqrt{p}}} G\left(-\frac{1}{p \tau}\right)\left(-\frac{z}{p \tau}-\frac{1}{p}\right)^{k-2} \frac{1}{p \tau^{2}} d \tau \\
& =(\sqrt{p} z)^{k-2} \int_{\frac{1}{p z}}^{\zeta / \sqrt{p}} G(\tau)\left(\tau-\frac{1}{p z}\right)^{k-2} d \tau=\left(\left.\Phi_{G}^{+}\right|_{2-k} W_{p}\right)(z) .
\end{aligned}
$$

For (2) we have from (1) that

$$
\begin{aligned}
H_{G}^{+}(-z)= & \int_{-\frac{1}{\sqrt{p} \zeta}}^{\zeta / \sqrt{p}} G(\tau)(z+\tau)^{k-2} d \tau=\int_{-\bar{z}}^{\zeta / \sqrt{p}} G(\tau)(z+\tau)^{k-2} d \tau \\
& +\int_{-\frac{1}{\sqrt{p} \zeta}}^{-\bar{z}} G(\tau)(z+\tau)^{k-2} d \tau \\
= & \left(\left.\Phi_{G}^{+}\right|_{2-k}\left(1-T^{-1}\right)\right)(z)=\left(\left.\Phi_{G}^{+}\right|_{2-k}\left(1-W_{p}\right)\right)(z) .
\end{aligned}
$$

In the next proposition we deal with the surjectivity problem of the differential operator $\xi_{2-k}$.

Proposition 3.3. (1) $\xi_{2-k}: H_{2-k}\left(\Gamma_{0}^{+}(p)\right) \rightarrow M_{k}^{!}\left(\Gamma_{0}^{+}(p)\right)$ is surjective.

(2) $\xi_{2-k}: H_{2-k}^{*}\left(\Gamma_{0}^{+}(p)\right) \rightarrow S_{k}\left(\Gamma_{0}^{+}(p)\right)$ is surjective.

Proof. First we note that the differential operator $\xi_{2-k}$ defines an antilinear surjective map from $H_{2-k}\left(\Gamma_{0}(p)\right)$ (resp. $\left.H_{2-k}^{*}\left(\Gamma_{0}(p)\right)\right)$ to $M_{k}^{!}\left(\Gamma_{0}(p)\right)\left(\operatorname{resp} . S_{k}\left(\Gamma_{0}(p)\right)\right)$ (see [2, p.405]). Since

$$
\xi_{2-k}\left(\left.f\right|_{2-k} \gamma\right)=\left.\left(\xi_{2-k} f\right)\right|_{k} \gamma
$$

for any function $f$ on $\mathbb{H}$ and $\gamma \in S L_{2}(\mathbb{R})$, the differential operator $\xi_{2-k}$ defines maps $\xi_{2-k}: H_{2-k}\left(\Gamma_{0}^{+}(p)\right) \rightarrow M_{k}^{!}\left(\Gamma_{0}^{+}(p)\right)$ and $\xi_{2-k}: H_{2-k}^{*}\left(\Gamma_{0}^{+}(p)\right) \rightarrow S_{k}\left(\Gamma_{0}^{+}(p)\right)$. Let $f \in M_{k}^{!}\left(\Gamma_{0}^{+}(p)\right)$. Then there exists $g \in H_{2-k}\left(\Gamma_{0}(p)\right)$ such that $\xi_{2-k} g=f$. We then have

$$
\xi_{2-k}\left(\left(g+\left.g\right|_{2-k} W_{p}\right) / 2\right)=\left(\xi_{2-k} g\right) / 2+\left(\left.\left(\xi_{2-k} g\right)\right|_{k} W_{p}\right) / 2=f .
$$

Since $g+\left.g\right|_{2-k} W_{p} \in H_{2-k}\left(\Gamma_{0}^{+}(p)\right)$, the first assertion holds. Now let $f \in S_{k}\left(\Gamma_{0}^{+}(p)\right)$. Then there exists $h \in H_{2-k}^{*}\left(\Gamma_{0}(p)\right)$ such that $\xi_{2-k} h=f$. The same argument as in the previous case allows us to show that $\xi_{2-k}\left(\left(h+\left.h\right|_{2-k} W_{p}\right) / 2\right)=f$. Moreover, since

$$
\xi_{2-k}\left(\left.h\right|_{2-k} W_{p}\right)=\left.\left(\xi_{2-k} h\right)\right|_{k} W_{p}=f \in S_{k}\left(\Gamma_{0}^{+}(p)\right) \subseteq S_{k}\left(\Gamma_{0}(p)\right),
$$

we observe that $\left.h\right|_{2-k} W_{p} \in H_{2-k}^{*}\left(\Gamma_{0}(p)\right)$, and hence $h+\left.h\right|_{2-k} W_{p} \in H_{2-k}^{*}\left(\Gamma_{0}^{+}(p)\right)$.

Suppose that $G \in S_{k}^{!}\left(\Gamma_{0}^{+}(p)\right)$. Then there exists a harmonic weak Maass form $\mathcal{F} \in H_{2-k}\left(\Gamma_{0}^{+}(p)\right)$ such that $\xi_{2-k}(\mathcal{F})(z)=(2 i)^{k-1} G^{c}(z)$. We can easily show that

$$
\mathcal{F}(z)=\int_{-\bar{z}}^{-\frac{1}{\sqrt{p} \zeta}} G(\tau)(z+\tau)^{k-2} d \tau+C_{G}^{+}(z)
$$

for some holomorphic function $C_{G}^{+}(z)$ on $\mathbb{H}$. In the next proposition we investigate the relationship between $\Phi_{G}^{+}(z)$ and $C_{G}^{+}$. 
Proposition 3.4. Let $p=2$ or 3. For the functions $\Phi_{G}^{+}(z)$ and $C_{G}^{+}$defined in (4) and (5) we have that

(1) $\Phi_{G}^{+}(z)=\mathcal{F}(z)-\left(\left.C_{G}^{+}\right|_{2-k} T\right)(z)=\mathcal{F}(z)-\left(\left.C_{G}^{+}\right|_{2-k} W_{p}\right)(z)$,

(2) $\left(\left.C_{G}^{+}\right|_{2-k} T\right)(z)=\left(\left.C_{G}^{+}\right|_{2-k} W_{p}\right)(z)$.

Proof. For (1) we have from Proposition 3.2 that

$\mathcal{F}(z)-C_{G}^{+}(z)=\int_{-\bar{z}}^{-\frac{1}{\sqrt{p} \zeta}} G(\tau)(z+\tau)^{k-2} d \tau=\left(\left.\Phi_{G}^{+}\right|_{k-2} T^{-1}\right)(z)=\left(\left.\Phi_{G}^{+}\right|_{k-2} W_{p}\right)(z)$,

which implies the first assertion. (2) follows from (1).

Proposition 3.5. For any polynomial $p(z)$ of degree at most $-l \in 2 \mathbb{N}$, let $\widetilde{p}(z)=$ $p(-z)$. Then we have that $\left.\widetilde{\left(\left.p\right|_{l} W_{p}\right.}\right)(z)=\left(\left.\widetilde{p}\right|_{l} W_{p}\right)(z)$ and $\left(\widetilde{\left.p\right|_{l} T}\right)(z)=\left(\left.\widetilde{p}\right|_{l} T^{-1}\right)(z)$.

Proof. We can show the assertion by direct calculation.

Let

$$
E_{k}(z)=1-\frac{2 k}{B_{k}} \sum_{n=1}^{\infty} \sigma_{k-1}(n) q^{n}, \quad E_{k}^{+}=E_{k, p}^{+}(z)=\frac{E_{k}(z)+p^{k / 2} E_{k}(p z)}{1+p^{k / 2}} .
$$

Here we note that $E_{k}^{+}$is a modular form of weight $k$ for $\Gamma_{0}^{+}(p)$ with a nonzero Fourier constant coefficient.

Proposition 3.6. We have that

$$
\overline{r^{+}\left(E_{k}^{+} ; \bar{z}\right)} \equiv r^{+}\left(E_{k}^{+} ; z\right) \quad\left(\bmod (\sqrt{p} z)^{k-2}-1\right) .
$$

Proof. We can easily show that

$$
\mathcal{E}_{E_{k}}-\left.p^{k / 2} \mathcal{E}_{E_{k}(p z)}\right|_{2-k} W_{p}=\mathcal{E}_{E_{k}}-\left.\mathcal{E}_{E_{k}}\right|_{2-k} S=c_{k}^{-1} r\left(E_{k} ; z\right),
$$

and hence we obtain that

$$
\begin{aligned}
\left(1+\sqrt{p}^{k}\right) r^{+}\left(E_{k}^{+} ; z\right) & =\left(1+\sqrt{p}^{k}\right) c_{k}\left(\mathcal{E}_{E_{k}^{+}}-\left.\mathcal{E}_{E_{k}^{+}}\right|_{2-k} W_{p}\right) \\
& =c_{k}\left(\mathcal{E}_{E_{k}}+p^{k / 2} \mathcal{E}_{E_{k}(p z)}-\left.\mathcal{E}_{E_{k}}\right|_{2-k} W_{p}-\left.p^{k / 2} \mathcal{E}_{E_{k}(p z)}\right|_{2-k} W_{p}\right) \\
& =c_{k}\left(p^{k / 2} \mathcal{E}_{E_{k}(p z)}-\left.\mathcal{E}_{E_{k}}\right|_{2-k} W_{p}\right)+r\left(E_{k} ; z\right) \\
& =-\left.r\left(E_{k} ; z\right)\right|_{2-k} W_{p}+r\left(E_{k} ; z\right) .
\end{aligned}
$$

On the other hand, from [2, (1.9) and (4.4)] we have that

$$
\begin{aligned}
r\left(E_{k} ; z\right)= & \frac{1}{k-1}\left(z^{k-1}+\frac{1}{z}\right)+\sum_{\substack{0<n<k-2 \\
n: \text { odd }}} i^{1-n}(\underset{n}{k-2}) r_{n}\left(E_{k}\right) z^{k-2-n} \\
& +i r_{0}\left(E_{k}\right)\left(z^{k-2}-1\right)
\end{aligned}
$$

and

$$
\begin{aligned}
\left.r\left(E_{k} ; z\right)\right|_{2-k} W_{p}= & -\frac{p^{-k / 2}}{(k-1) z}-\frac{p^{k / 2} z^{k-1}}{(k-1)}-\sum_{\substack{0<n<k-2 \\
n: \text { odd }}} i^{1-n}\left(\begin{array}{c}
k-2 \\
n
\end{array}\right) r_{n}\left(E_{k}\right) p^{n-k / 2+1} z^{n} \\
& +i r_{0}\left(E_{k}\right)\left(\sqrt{p}^{2-k}-(\sqrt{p} z)^{k-2}\right) .
\end{aligned}
$$


Consequently from (6), (7) and (8) we have that

$$
\begin{aligned}
\left(1+\sqrt{p}^{k}\right) r^{+}\left(E_{k}^{+} ; z\right)= & \frac{1+\sqrt{p}^{-k}}{(k-1) z}+\frac{\left(1+\sqrt{p}^{k}\right) z^{k-1}}{k-1}+\sum_{\substack{0<n<k-2 \\
n: \text { odd }}} \alpha_{n} z^{n} \\
& +i r_{0}\left(E_{k}\right)\left(1+\sqrt{p}^{2-k}\right)\left((\sqrt{p} z)^{k-2}-1\right)
\end{aligned}
$$

for some real numbers $\alpha_{n}$, and hence

$$
\overline{r^{+}\left(E_{k}^{+} ; \bar{z}\right)}-r^{+}\left(E_{k}^{+} ; z\right)=\left(\overline{i r_{0}\left(E_{k}\right)}-i r_{0}\left(E_{k}\right)\right)\left(\frac{1+\sqrt{p}^{2-k}}{1+\sqrt{p}^{k}}\right)\left((\sqrt{p} z)^{k-2}-1\right),
$$

which implies the assertion.

We now define the Poincaré series and Maass-Poincaré series for $\Gamma_{0}^{+}(p)$ as follows:

$$
\begin{aligned}
P^{+}(0, k, p ; z) & :=P(0, k, 1, p ; z)+\left.P(0, k, 1, p ; z)\right|_{k} W_{p}, \\
F^{+}(0,2-k, p ; z) & :=P\left(0,2-k,(4 \pi y)^{k-1}, p ; z\right)+\left.P\left(0,2-k,(4 \pi y)^{k-1}, p ; z\right)\right|_{2-k} W_{p},
\end{aligned}
$$

where $P(m, k, \varphi, p ; z)$ stands for the sum

$$
P(m, k, \varphi, p ; z)=\sum_{\gamma \in \Gamma_{\infty} \backslash \Gamma_{0}(p)}\left(\left.(\varphi(y) e(m x))\right|_{k} \gamma\right)(z) .
$$

We then observe that $E_{k}^{+}(z)=P^{+}(0, k, p ; z)$ (see [11, p. 488]) and $F^{+}(0,2-k, p ; z) \in$ $H_{2-k}\left(\Gamma_{0}^{+}(p)\right)$ (see [3, p. 9]). In the following proposition we compute the images of $F^{+}(0,2-k, p ; z)$ under the differential operators $\xi_{2-k}$ and $D^{k-1}$.

Proposition 3.7. We have that

$$
\xi_{2-k}\left(F^{+}(0,2-k, p ; z)\right)=(k-1)(4 \pi)^{k-1} E_{k}^{+}(z)
$$

and

$$
D^{k-1}\left(F^{+}(0,2-k, p ; z)\right)=-\Gamma(k) E_{k}^{+}(z) .
$$

Proof. From [3, Lemma 2.3] we have that

$$
\begin{aligned}
\xi_{2-k}\left(F^{+}(0,2-k, p ; z)\right) & =\xi_{2-k}(F(0,2-k, p ; z))+\xi_{2-k}\left(\left.F(0,2-k, p ; z)\right|_{2-k} W_{p}\right) \\
& =\xi_{2-k}(F(0,2-k, p ; z))+\left.\xi_{2-k}(F(0,2-k, p ; z))\right|_{k} W_{p} \\
& =(k-1)(4 \pi)^{k-1}\left(P(0, k, p ; z)+\left.P(0, k, p ; z)\right|_{k} W_{p}\right) \\
& =(k-1)(4 \pi)^{k-1} P^{+}(0, k, p ; z)=(k-1)(4 \pi)^{k-1} E_{k}^{+}
\end{aligned}
$$

and

$$
\begin{aligned}
D^{k-1}\left(F^{+}(0,2-k, p ; z)\right) & =D^{k-1}(F(0,2-k, p ; z))+D^{k-1}\left(\left.F(0,2-k, p ; z)\right|_{2-k} W_{p}\right) \\
& =D^{k-1}(F(0,2-k, p ; z))+\left.\left(D^{k-1}(F(0,2-k, p ; z))\right)\right|_{k} W_{p} \\
& =-\Gamma(k)\left(P(0, k, p ; z)+\left.P(0, k, p ; z)\right|_{k} W_{p}\right) \\
& =-\Gamma(k) P^{+}(0, k, p ; z)=-\Gamma(k) E_{k}^{+} .
\end{aligned}
$$

From Proposition 3.6 and Proposition 3.7 we obtain the following theorem, which gives period relations for the Eisenstein series. 
Theorem 3.8. We have that

$$
\begin{aligned}
& r^{+}\left(\xi_{2-k}\left(F^{+}(0,2-k, p ; z)\right) ; z\right) \\
& \quad \equiv \frac{-(4 \pi)^{k-1}}{\Gamma(k-1)} \overline{r^{+}\left(D^{k-1}\left(F^{+}(0,2-k, p ; z)\right) ; \bar{z}\right)} \quad\left(\bmod (\sqrt{p} z)^{k-2}-1\right) .
\end{aligned}
$$

Now we are ready to prove Theorem 1.3. For a fixed $G \in S_{k}^{!}\left(\Gamma_{0}^{+}(p)\right)$, we assume that $\mathcal{F} \in H_{2-k}\left(\Gamma_{0}^{+}(p)\right)$ satisfies $\xi_{2-k}(\mathcal{F})=(2 i)^{k-1} G^{c}(z)$. Let $F:=D^{k-1}(\mathcal{F})$. Then $D^{k-1}\left(\mathcal{E}_{F}^{+}(z)\right)=F(z)$. Moreover we have that $\widetilde{r^{+}(F ; z)} \equiv-c_{k} r^{+}(G ; z)$ $\left(\bmod (\sqrt{p} z)^{k-2}-1\right)$. Indeed, let $p_{G}^{+}(z):=C_{G}^{+}(z)-\mathcal{E}_{F}^{+}(z)$. Then $D^{k-1}\left(p_{G}^{+}(z)\right)=0$, and hence $p_{G}^{+}(z)$ is a polynomial of degree at most $k-2$. We have that

$$
\begin{aligned}
\left(\left.p_{G}^{+}\right|_{2-k}\left(1-W_{p}\right)\right)(z) & =\left(\left.C_{G}^{+}\right|_{2-k}\left(1-W_{p}\right)\right)(z)-\left(\left.\mathcal{E}_{F}^{+}\right|_{2-k}\left(1-W_{p}\right)\right)(z) \\
& =\left(\left.C_{G}^{+}\right|_{2-k}\left(1-W_{p}\right)\right)(z)-\frac{1}{c_{k}} r^{+}(F ; z)
\end{aligned}
$$

and

$$
\begin{aligned}
\left(\left.p_{G}^{+}\right|_{2-k}(1-T)\right)(z) & =\left(\left.C_{G}^{+}\right|_{2-k}(1-T)\right)(z)-\left(\left.\mathcal{E}_{F}^{+}\right|_{2-k}(1-T)\right)(z) \\
& =\left(\left.C_{G}^{+}\right|_{2-k}(1-T)\right)(z) .
\end{aligned}
$$

From (9), (10) and Proposition 3.4 we obtain that for $p=2$ or 3 ,

$$
\begin{aligned}
\frac{1}{c_{k}} r^{+}(F ; z) & =\left(\left.C_{G}^{+}\right|_{2-k}\left(1-W_{p}\right)\right)(z)-\left(\left.p_{G}^{+}\right|_{2-k}\left(1-W_{p}\right)\right)(z) \\
& =\left(\left.C_{G}^{+}\right|_{2-k}(1-T)\right)(z)-\left(\left.p_{G}^{+}\right|_{2-k}\left(1-W_{p}\right)\right)(z) \\
& =\left(\left.p_{G}^{+}\right|_{2-k}(1-T)\right)(z)-\left(\left.p_{G}^{+}\right|_{2-k}\left(1-W_{p}\right)\right)(z) \\
& =\left(\left.p_{G}^{+}\right|_{2-k}\left(W_{p}-T\right)\right)(z) .
\end{aligned}
$$

We now relate the polynomials $H_{G}^{+}$and $P_{G}^{+}$. We have from (10), Proposition 3.2 and Proposition 3.4, that

$$
\begin{aligned}
\widetilde{H_{G}^{+}}(z) & =H_{G}^{+}(-z)=\left(\left.\Phi_{G}^{+}\right|_{2-k}\left(1-T^{-1}\right)\right)(z)=\left(\left.\left(\mathcal{F}-\left.C_{G}^{+}\right|_{2-k} T\right)\right|_{2-k}\left(1-T^{-1}\right)\right)(z) \\
& =\left(\left.\mathcal{F}\right|_{2-k}\left(1-T^{-1}\right)\right)(z)-\left.C_{G}^{+}\right|_{2-k}(T-1)(z)=\left.C_{G}^{+}\right|_{2-k}(1-T)(z) \\
& =\left(\left.P_{G}^{+}\right|_{2-k}(1-T)\right)(z),
\end{aligned}
$$

and hence from Proposition 3.5 we have that

$$
\begin{aligned}
& \left.H_{G}^{+}(z)=\widetilde{\widetilde{H_{G}^{+}}}(z)=\left(\left.P_{G}^{+}\right|_{2-k}(1-T)\right)(z)=\widetilde{P_{G}^{+}}(z)-\widetilde{\left(\left.P_{G}^{+}\right|_{2-k} T\right.}\right)(z) \\
& \left.\left.=\widetilde{P_{G}^{+}}(z)-\left.\widetilde{\left(P_{G}^{+}\right.}\right|_{2-k} T^{-1}\right)(z)=\left.\widetilde{\left(P_{G}^{+}\right.}\right|_{2-k} T^{-1}(T-1)\right)(z) \text {. }
\end{aligned}
$$

Now from Proposition 3.1 and (11) we have that

$$
\begin{aligned}
& \left.\left(\left.\left(q_{G}^{+}+\left.\widetilde{P_{G}^{+}}\right|_{2-k} T^{-1}\right)\right|_{2-k}(1-T)\right)(z)=\left(\left.q_{G}^{+}\right|_{2-k}(1-T)\right)(z)+\left.\widetilde{\left(P_{G}^{+}\right.}\right|_{2-k} T^{-1}(1-T)\right)(z) \\
& =H_{G}^{+}(z)-H_{G}^{+}(z)=0 \text {, }
\end{aligned}
$$

which means that the polynomial $\left.\left(q_{G}^{+}+\left.\widetilde{P_{G}^{+}}\right|_{2-k} T^{-1}\right)\right)(z)$ is a constant, say $\alpha$. Applying $T W_{p}$ to the resulting identity

$$
\left.\left.q_{G}^{+}(z)=-\left.\widetilde{\left(P_{G}^{+}\right.}\right|_{2-k} T^{-1}\right)\right)(z)+\alpha
$$

we obtain

$$
\left.\left(\left.q_{G}^{+}\right|_{2-k} T W_{p}\right)(z)=-\left.\widetilde{\left(P_{G}^{+}\right.}\right|_{2-k} W_{p}\right)(z)+\alpha(\sqrt{p} z)^{k-2} .
$$


We now compare $c_{k} r^{+}(G ; z)$ and $\widetilde{r^{+(F ; z)}}$. By Proposition 3.5 and the fact that $c_{k}^{-1} r^{+}(F ; z)=\left(\left.P_{G}^{+}\right|_{2-k}\left(W_{p}-T\right)\right)(z)$, we have

$$
\left.c_{k}^{-1} r^{+(F ; z)}=\left(\left.P_{G}^{+}\right|_{2-k} \widetilde{\left(W_{p}-T\right)}\right)(z)=\left.\widetilde{\left(P_{G}^{+}\right.}\right|_{2-k}\left(W_{p}-T^{-1}\right)\right)(z),
$$

and hence from Proposition 3.1 we have that

$$
\begin{aligned}
c_{k}^{-1} r^{+(F ; z)}+r^{+}(G ; z)= & \left.\left.\left.\widetilde{\left(P_{G}^{+}\right.}\right|_{2-k} W_{p}\right)(z)-\left.\widetilde{\left(P_{G}^{+}\right.}\right|_{2-k} T^{-1}\right)(z)+\left(\left.q_{G}^{+}\right|_{2-k} W_{p}\right)(z) \\
& -\left(\left.q_{G}^{+}\right|_{2-k} T\right)(z) \\
= & -\left(\left.q_{G}^{+}\right|_{2-k} T W_{p}\right)(z)+\alpha\left((\sqrt{p} z)^{k-2}-1\right)+q_{G}^{+}(z) \\
& +\left(\left.q_{G}^{+}\right|_{2-k} W_{p}\right)(z)-\left(\left.q_{G}^{+}\right|_{2-k} T\right)(z) \\
= & \left(\left.q_{G}^{+}\right|_{2-k}(1-T)\left(1+W_{p}\right)\right)(z)+\alpha\left((\sqrt{p} z)^{k-2}-1\right) .
\end{aligned}
$$

Since Proposition 3.1 gives

$$
\left(\left.q_{G}^{+}\right|_{2-k}(1-T)\right)(z)=H_{G}^{+}(z) \text { and }\left(\left.H_{G}^{+}\right|_{2-k}\left(1+W_{p}\right)\right)(z)=0,
$$

we conclude that

$$
0=\left(\left.\left(\left.q_{G}^{+}\right|_{2-k}(1-T)\right)\right|_{2-k}\left(1+W_{p}\right)\right)(z)=\left(\left.q_{G}^{+}\right|_{2-k}(1-T)\left(1+W_{p}\right)\right)(z),
$$

and hence we see that

$$
\widetilde{r^{+}(F ; z)} \equiv-c_{k} r^{+}(G ; z) \quad\left(\bmod (\sqrt{p} z)^{k-2}-1\right) .
$$

Noticing that $\left.r^{+}\left(G^{c} ; z\right)=-\overline{r^{+}(G ;-\bar{z}}\right), F=D^{k-1} \mathcal{F}$ and $\xi_{2-k}(\mathcal{F})=(2 i)^{k-1} G^{c}(z)$, we obtain from (12) that

$$
r^{+}\left(\xi_{2-k}(\mathcal{F}) ; z\right) \equiv-\frac{(4 \pi)^{k-1}}{\Gamma(k-1)} \overline{r^{+}\left(D^{k-1} \mathcal{F} ; \bar{z}\right)} \quad\left(\bmod (\sqrt{p} z)^{k-2}-1\right)
$$

Consequently the first assertion holds in the case $G \in S_{k}^{!}\left(\Gamma_{0}^{+}(p)\right)$, and from Theorem 3.8 we complete a proof of the first assertion. To prove the second assertion, take a weakly holomorphic modular form $\mathcal{W} \in M_{2-k}^{!}\left(\Gamma_{0}^{+}(p)\right)$ with Fourier expansion $\mathcal{W}(z)=\sum_{n \gg-\infty} a_{n} q^{n}$ with $a_{0} \neq 0$. Such a form exists. Indeed, take a weakly holomorphic modular form $f \in M_{2-k}^{!}\left(\Gamma_{0}(p)\right)$ with Fourier expansion $f(z)=\sum_{n \gg-\infty} b_{n} q^{n}$ with $b_{0} \neq 0$ (see [2, Proposition 3.5]); then we can take $\mathcal{W}(z)$ by $f(z)+p^{k / 2} f(p z)$. We note that $r^{+}\left(D^{k-1} \mathcal{W} ; z\right)=a_{0} c_{k}\left((\sqrt{p} z)^{k-2}-1\right)$. Since $\xi_{2-k}(\mathcal{W})=0$ and $r^{+}\left(D^{k-1} \mathcal{F} ; z\right)-r^{+}\left(D^{k-1}(\mathcal{F}+\mathcal{W}) ; z\right)=-a_{0} c_{k}\left((\sqrt{p} z)^{k-2}-1\right)$, noticing that

$$
r^{+}\left(\xi_{2-k}(\mathcal{F}) ; z\right)+\frac{(4 \pi)^{k-1}}{\Gamma(k-1)} \overline{r^{+}\left(D^{k-1}(\mathcal{F}) ; \bar{z}\right)}=\beta\left((\sqrt{p} z)^{k-2}-1\right) \text { for some } \beta \in \mathbb{C},
$$

we obtain that

$$
\begin{aligned}
r^{+}\left(\xi_{2-k}(\mathcal{F}) ; z\right) & =-\frac{(4 \pi)^{k-1}}{\Gamma(k-1)} \overline{r^{+}\left(D^{k-1}(\mathcal{F}) ; \bar{z}\right)}+\beta c_{k}\left((\sqrt{p} z)^{k-2}-1\right) \\
& =-\frac{(4 \pi)^{k-1}}{\Gamma(k-1)} \overline{r^{+}\left(D^{k-1}(\mathcal{F}+\mathcal{W}) ; \bar{z}\right)}+\left(\beta+\overline{a_{0} c_{k}}\right)\left((\sqrt{p} z)^{k-2}-1\right)
\end{aligned}
$$


If $\beta=0$, then the second assertion in Theorem 1.3 holds. If $\beta \neq 0$, then take $\mathcal{W}$ such that $a_{0} c_{k}=-\bar{\beta}$. Then we get

$$
r^{+}\left(\xi_{2-k}(\mathcal{F}) ; z\right)=-\frac{(4 \pi)^{k-1}}{\Gamma(k-1)} \overline{r^{+}\left(D^{k-1}(\widehat{\mathcal{F}}) ; \bar{z}\right)}
$$

by taking $\widehat{\mathcal{F}}=\mathcal{F}+\mathcal{W} \in H_{2-k}\left(\Gamma_{0}^{+}(p)\right)$.

\section{Proof of Theorem 1.5}

First we begin with finding a relation between the period function of a cusp form and the critical values of an $L$-function of the cusp form.

Proposition 4.1. Suppose that $A(z)=\sum_{n=1}^{\infty} a(n) q^{n}$ and $B(z)=\sum_{n=1}^{\infty} b(n) q^{n}$ are holomorphic functions on $\mathbb{H}$ where $|a(n)|,|b(n)|=O\left(n^{\delta}\right)$ and $\delta>0$. If

$$
A(z)=\left(\left.B\right|_{k} W_{p}\right)(z)
$$

where $k \geq 2$ is even, then

$$
\mathbb{E}_{A, k}(z)-\left(\left.\mathbb{E}_{B, k}\right|_{2-k} W_{p}\right)(z)=\sum_{j=0}^{k-2} \frac{L(A,-j+k-1)}{j !}(2 \pi i z)^{j} .
$$

Here $L(A, s)$ is the analytic continuation of

$$
L(A, s):=\sum_{n=1}^{\infty} \frac{a(n)}{n^{s}}
$$

and

$$
\mathbb{E}_{\phi, k}(z):=\sum_{n=1}^{\infty} \nu(n) n^{1-k} q^{n},
$$

where $\phi(z)=\sum_{n=1}^{\infty} \nu(n) q^{n}$.

Proof. Since $A(z)=\left(\left.B\right|_{k} W_{p}\right)(z)$, the analytically continued Dirichlet series for $A(z)$ and $B(z)$, say $L(A, s)$ and $L(B, s)$, satisfy the functional equation (see [9])

$$
\Lambda_{A}(s)=i^{k} \Lambda_{B}(k-s),
$$

which gives

$$
\widehat{\Lambda}_{A}(s)=-i^{k} \widehat{\Lambda}_{B}(2-k-s) .
$$

Here

$$
\begin{aligned}
\Lambda_{A}(s) & :=\left(\frac{2 \pi}{\sqrt{p}}\right)^{-s} \Gamma(s) L(A, s), \\
\Lambda_{B}(s) & :=\left(\frac{2 \pi}{\sqrt{p}}\right)^{-s} \Gamma(s) L(B, s), \\
L(A, s) & :=\sum_{n=1}^{\infty} \frac{a(n)}{n^{s}}, \quad L(B, s):=\sum_{n=1}^{\infty} \frac{b(n)}{n^{s}}, \\
\widehat{\Lambda}_{A}(s) & :=\left(\frac{2 \pi}{\sqrt{p}}\right)^{-s} \Gamma(s) L(A, s+k-1), \\
\widehat{\Lambda}_{B}(s) & :=\left(\frac{2 \pi}{\sqrt{p}}\right)^{-s} \Gamma(s) L(B, s+k-1) .
\end{aligned}
$$


We have from (13) and (14) that

$$
\widehat{\Lambda}_{A}(s)=\Lambda_{A}(s+k-1)\left(\frac{2 \pi}{\sqrt{p}}\right)^{k-1} \frac{1}{s(s+1) \cdots(s+k-2)} .
$$

Since $\Lambda_{A}(s)$ is entire and bounded in a vertical strips (see [9, Theorem 7.3]), we obtain that

$$
\widehat{\Lambda}_{A}(s)-\left(\frac{2 \pi}{\sqrt{p}}\right)^{k-1} \sum_{r=2-k}^{0} \frac{a_{r}}{s-r}
$$

is entire and bounded in a vertical strip, where

$$
a_{r}=\operatorname{Res}_{s=r} \frac{\Lambda_{A}(s+k-1)}{s(s+1) \cdots(s+k-2)} .
$$

Using the Mellin inversion formula [13, Section 9.3], we have that for $c_{1}>0$ (which we will choose sufficiently small),

$$
\begin{aligned}
& \mathbb{E}_{A, k}(z)=\frac{1}{2 \pi i} \int_{c_{1}-i \infty}^{c_{1}+i \infty} \widehat{\Lambda}_{A}(s)\left(\frac{\sqrt{p} z}{i}\right)^{-s} d s, \\
& \mathbb{E}_{B, k}(z)=\frac{1}{2 \pi i} \int_{c_{1}-i \infty}^{c_{1}+i \infty} \widehat{\Lambda}_{B}(s)\left(\frac{\sqrt{p} z}{i}\right)^{-s} d s .
\end{aligned}
$$

We note that from (14) we have

$$
\begin{aligned}
\mathbb{E}_{B, k}(z) & =-\frac{1}{2 \pi i} \int_{2-k-c_{1}+i \infty}^{2-k-c_{1}-i \infty} \widehat{\Lambda}_{B}(2-k-s)\left(\frac{\sqrt{p} z}{i}\right)^{s+k-2} d s \\
& =\frac{1}{2 \pi i} \int_{2-k-c_{1}+i \infty}^{2-k-c_{1}-i \infty} i^{k} \widehat{\Lambda}_{A}(s)\left(\frac{\sqrt{p} z}{i}\right)^{s+k-2} d s,
\end{aligned}
$$

which gives

$$
-\left(\left.\mathbb{E}_{B, k}\right|_{2-k} W_{p}\right)(z)=\frac{1}{2 \pi i} \int_{2-k-c_{1}+i \infty}^{2-k-c_{1}-i \infty} \widehat{\Lambda}_{A}(s)\left(\frac{\sqrt{p} z}{i}\right)^{-s} d s .
$$

Consequently, since

$$
\widehat{\Lambda}_{A}(s)\left(\frac{\sqrt{p} z}{i}\right)^{-s}-\sum_{r=2-k}^{0} \frac{a_{r}}{s-r}\left(\frac{2 \pi}{\sqrt{p}}\right)^{k-1}\left(\frac{\sqrt{p} z}{i}\right)^{-s}
$$


is entire, we obtain

$$
\begin{aligned}
& \mathbb{E}_{A, k}(z)-\left(\left.\mathbb{E}_{B, k}\right|_{2-k} W_{p}\right)(z) \\
= & \frac{1}{2 \pi i} \int_{c_{1}-i \infty}^{c_{1}+i \infty} \widehat{\Lambda}_{A}(s)\left(\frac{\sqrt{p} z}{i}\right)^{-s} d s+\frac{1}{2 \pi i} \int_{2-k-c_{1}+i \infty}^{2-k-c_{1}-i \infty} \widehat{\Lambda}_{A}(s)\left(\frac{\sqrt{p} z}{i}\right)^{-s} d s \\
= & \sum_{r=2-k}^{0} \operatorname{Res}_{s=r}\left(\frac{a_{r}}{s-r}\left(\frac{2 \pi}{\sqrt{p}}\right)^{k-1}\left(\frac{\sqrt{p} z}{i}\right)^{-s}\right) \text { by Cauchy Residue Theorem } \\
= & \left(\frac{2 \pi}{\sqrt{p}}\right)^{k-1} \sum_{r=2-k}^{0} a_{r}\left(\frac{\sqrt{p} z}{i}\right)^{-r}=\left(\frac{2 \pi}{\sqrt{p}}\right)^{k-1} \sum_{r=0}^{k-2} a_{-r}\left(\frac{\sqrt{p} z}{i}\right)^{r} \\
= & \sum_{r=0}^{k-2} \frac{\Gamma(-r+k-1) L(A,-r+k-1)}{(-r)(-r+1) \cdots(-1) 1 \cdots(-r+k-2)}(-2 \pi i z)^{r} \\
= & \sum_{r=0}^{k-2} \frac{L(A,-j+k-1)}{r !}(2 \pi i z)^{r} .
\end{aligned}
$$

By Proposition 4.1 we obtain the following theorem which relates the period polynomials of cusp forms to their critical $L$-values.

Theorem 4.2. Let $f \in S_{k}\left(\Gamma_{0}^{+}(p)\right)$. Then

$$
c_{k}^{-1} r^{+}(f ; z)=\sum_{n=0}^{k-2} \frac{L(f, n+1)}{(k-2-n) !}(2 \pi i z)^{k-2-n} .
$$

For each $f \in S_{k}\left(\Gamma_{0}^{+}(p)\right)$ we define the $n$th period of $f$ by

$$
r_{n}(f):=\int_{0}^{\infty} f(i t) t^{n} d t \quad(0 \leq n \leq k-2) .
$$

Define two period maps $r_{ \pm}: S_{k}\left(\Gamma_{0}^{+}(p)\right) \rightarrow P_{k-2}$ by

$$
\begin{aligned}
r_{+}(f ; z) & :=\sum_{\substack{0 \leq n \leq k-2 \\
n: \text { even }}}(-1)^{n / 2}\left(\begin{array}{c}
k-2 \\
n
\end{array}\right) r_{n}(f) z^{k-2-n}, \\
r_{-}(f ; z) & :=\sum_{\substack{0 \leq n \leq k-2 \\
n: \text { odd }}}(-1)^{(n-1) / 2}\left(\begin{array}{c}
k-2 \\
n
\end{array}\right) r_{n}(f) z^{k-2-n},
\end{aligned}
$$

where $P_{k-2}$ is the space of polynomials in $z$ of degree at most $k-2$ with coefficients in $\mathbb{C}$. Notice that if we let $r(f ; z):=r_{-}(f ; z)+i r_{+}(f ; z)$, then

$$
r(f ; z)=\sum_{0 \leq n \leq k-2} i^{-n+1}\left(\begin{array}{c}
k-2 \\
n
\end{array}\right) r_{n}(f) z^{k-2-n}=\int_{0}^{i \infty} f(\tau)(z-\tau)^{k-2} d \tau,
$$

and the critical $L$-values of the cusp form $f$ are expressed in terms of the period $r_{n}(f)$ as in the following lemma.

Lemma 4.3. For each $f \in S_{k}\left(\Gamma_{0}^{+}(p)\right)$ we have that

$$
L(f, n+1)=\frac{(2 \pi)^{n+1}}{n !} r_{n}(f),
$$

for all $0 \leq n \leq k-2$. 
Proof. Since $\int_{0}^{\infty} f(i t) t^{s} \frac{d t}{t}=(2 \pi)^{-s} \Gamma(s) L(f, s)$, we have from the definition of $r_{n}(f)$ the assertion.

The following corollary motivates the definition of the period function $r^{+}(f ; z)$.

Corollary 4.4. For $f \in S_{k}\left(\Gamma_{0}^{+}(p)\right)$ we have that

$$
r^{+}(f ; z)=r(f ; z) .
$$

Proof. By Theorem 4.2 and Lemma 4.3 we have the assertion.

Remark 4.5. It is well known [2, (1.8)] that $\mathcal{E}_{f}(z)$ has an integral representation, i.e.

$$
\mathcal{E}_{f}(z)=\frac{1}{c_{k}} \int_{z}^{i \infty} f(\tau)(z-\tau)^{k-2} d \tau .
$$

Using (15) one can also derive Corollary 4.4,

Noticing that $\mathcal{F}^{+}=\mathcal{E}_{D^{k-1} \mathcal{F}^{+}}+c_{\mathcal{F}}^{+}(0)$, where $c_{\mathcal{F}}^{+}(0)$ is a constant term in the $q$-expansion of $\mathcal{F}^{+}$, we obtain that

$$
\begin{aligned}
\mathbb{P}\left(\mathcal{F}^{+}, W_{p} ; z\right) & =\frac{(4 \pi)^{k-1}}{\Gamma(k-1)}\left\{\left(\mathcal{E}_{D^{k-1} \mathcal{F}}-\left.\mathcal{E}_{D^{k-1} \mathcal{F}}\right|_{2-k} W_{p}\right)(z)-c_{\mathcal{F}}^{+}(0)\left((\sqrt{p} z)^{k-2}-1\right)\right\} \\
& =\frac{(4 \pi)^{k-1}}{\Gamma(k-1)} c_{k}^{-1} r^{+}\left(D^{k-1} \mathcal{F} ; z\right)-\frac{(4 \pi)^{k-1}}{\Gamma(k-1)} c_{\mathcal{F}}^{+}(0)\left((\sqrt{p} z)^{k-2}-1\right),
\end{aligned}
$$

which implies from Theorem 1.3 and Theorem 4.2 that for $p=2$ or 3 ,

$$
\begin{aligned}
\overline{\mathbb{P}\left(\mathcal{F}^{+}, W_{p} ; \bar{z}\right)} & =-\frac{(4 \pi)^{k-1}}{\Gamma(k-1)} c_{k}^{-1} \overline{r^{+}\left(D^{k-1} \mathcal{F} ; \bar{z}\right)}-\frac{(4 \pi)^{k-1}}{\Gamma(k-1)} \overline{c_{\mathcal{F}}^{+}(0)}\left((\sqrt{p} z)^{k-2}-1\right) \\
& \equiv c_{k}^{-1} r^{+}\left(\xi_{2-k}(\mathcal{F}) ; z\right) \quad\left(\bmod (\sqrt{p} z)^{k-2}-1\right) \\
& \equiv \sum_{n=0}^{k-2} \frac{L(f, n+1)}{(k-2-n) !}(2 \pi i z)^{k-2-n} \quad\left(\bmod (\sqrt{p} z)^{k-2}-1\right) .
\end{aligned}
$$

Now we will show that the congruence (16) is indeed an equality for all primes $p$. For this purpose we will use the integral representation of the the non-holomorphic part $\mathcal{F}^{-}$. It is well known [10, Section 7.3] that $\mathcal{F}^{-}$is a period integral of the cusp form $f=\xi_{2-k}(\mathcal{F})$. More precisely, we have

$$
\mathcal{F}^{-}(z)=-(2 i)^{1-k} \int_{-\bar{z}}^{i \infty} \overline{f(-\bar{\tau})}(\tau+z)^{k-2} d \tau .
$$

Let $\mathcal{F}_{f}(z):=\int_{-\bar{z}}^{i \infty} \overline{f(-\bar{\tau})}(\tau+z)^{k-2} d \tau$. For each $\gamma=\left(\begin{array}{ll}a & b \\ c & d\end{array}\right) \in S L_{2}(\mathbb{R})$ we observe that

$$
\begin{aligned}
\left(\left.\mathcal{F}_{f}\right|_{2-k} \gamma\right)(z) & =(c z+d)^{k-2} \mathcal{F}_{f}(\gamma z)=(c z+d)^{k-2} \int_{-\overline{\gamma z}}^{i \infty} \overline{f(-\bar{\tau})}(\tau+\gamma z)^{k-2} d \tau \\
& =(c z+d)^{k-2} \int_{\gamma^{*}(-\bar{z})}^{i \infty} \overline{f(-\bar{\tau})}(\tau+\gamma z)^{k-2} d \tau \quad \text { with } \gamma^{*}=\left(\begin{array}{cc}
-a & b \\
c & -d
\end{array}\right) \\
& =(c z+d)^{k-2} \int_{-\bar{z}}^{\left(\gamma^{*}\right)^{-1}(i \infty)}(c \tau-d)^{k} \overline{f(-\bar{\tau})}\left(\gamma^{*} \tau+\gamma z\right)^{k-2}(c \tau-d)^{-2} d \tau \\
& =\int_{-\bar{z}}^{\left(\gamma^{*}\right)^{-1}(i \infty)} \overline{f(-\bar{\tau})}(\tau+z)^{k-2} d \tau
\end{aligned}
$$


and

$$
\begin{aligned}
\mathcal{F}_{f}(z)-\left(\left.\mathcal{F}_{f}\right|_{2-k} \gamma\right)(z) & =\int_{-\bar{z}}^{i \infty} \overline{f(-\bar{\tau})}(\tau+z)^{k-2} d \tau-\int_{-\bar{z}}^{\left(\gamma^{*}\right)^{-1}(i \infty)} \overline{f(-\bar{\tau})}(\tau+z)^{k-2} d \tau \\
& =\int_{\left(\gamma^{*}\right)^{-1}(i \infty)}^{i \infty} \overline{f(-\bar{\tau})}(\tau+z)^{k-2} d \tau
\end{aligned}
$$

Thus we obtain that

$$
\begin{aligned}
\mathcal{F}_{f}(z)-\left(\left.\mathcal{F}_{f}\right|_{2-k} W_{p}\right)(z) & =\int_{0}^{i \infty} \overline{f(-\bar{\tau})}(\tau+z)^{k-2} d \tau \\
& =\sum_{n=0}^{k-2}\left(\begin{array}{c}
k-2 \\
n
\end{array}\right)\left(\int_{0}^{i \infty} \overline{f(-\bar{\tau})} \tau^{n} d \tau\right) z^{k-2-n} \\
& =\sum_{n=0}^{k-2}\left(\begin{array}{c}
k-2 \\
n
\end{array}\right)\left(i^{n+1} \int_{0}^{\infty} \overline{f(i t)} t^{n+1} \frac{d t}{t}\right) z^{k-2-n} \\
& =\sum_{n=0}^{k-2}\left(\begin{array}{c}
k-2 \\
n
\end{array}\right)\left(i^{n+1}(2 \pi)^{-(n+1)} \Gamma(n+1) \overline{L(f, n+1)}\right) z^{k-2-n} .
\end{aligned}
$$

Now observing

$$
\begin{aligned}
\mathbb{P}\left(\mathcal{F}^{+}, W_{p} ; z\right) & =\frac{(4 \pi)^{k-1}}{\Gamma(k-1)}\left(\mathcal{F}^{+}-\left.\mathcal{F}^{+}\right|_{2-k} W_{p}\right)(z)=-\frac{(4 \pi)^{k-1}}{\Gamma(k-1)}\left(\mathcal{F}^{-}-\left.\mathcal{F}^{-}\right|_{2-k} W_{p}\right)(z) \\
& =\frac{(4 \pi)^{k-1}}{\Gamma(k-1)}(2 i)^{1-k}\left(\mathcal{F}_{f}-\left.\mathcal{F}_{f}\right|_{2-k} W_{p}\right)(z)
\end{aligned}
$$

and utilizing (18) we find that

$$
\mathbb{P}\left(\mathcal{F}^{+}, W_{p} ; z\right)=\sum_{n=0}^{k-2} \frac{\overline{L(f, n+1)}}{(k-2-n) !}(-2 \pi i z)^{k-n-2} .
$$

Now the assertion in Theorem 1.5 is immediate from (19) and Theorem 4.2

\section{ACKNOWLEDGMENTS}

The authors wish to express their thanks to the referee for valuable comments. They also would like to thank the KIAS (Korea Institute for Advanced Study) for its hospitality.

\section{REFERENCES}

[1] G. Bol, Invarianten linearer differentialgleichungen (German), Abh. Math. Sem. Univ. Hamburg 16 (1949), no. nos. 3-4, 1-28. MR0033411(11,437a)

[2] Kathrin Bringmann, Pavel Guerzhoy, Zachary Kent, and Ken Ono, Eichler-Shimura theory for mock modular forms, Math. Ann. 355 (2013), no. 3, 1085-1121, DOI 10.1007/s00208012-0816-y. MR 3020155

[3] K. Bringmann, B. Kane and R. C. Rhoades, Duality and differential operators for harmonic weak Maass forms, Dev. Math., 28, Springer, New York, 2013. MR2986955

[4] Jan Hendrik Bruinier and Jens Funke, On two geometric theta lifts, Duke Math. J. 125 (2004), no. 1, 45-90, DOI 10.1215/S0012-7094-04-12513-8. MR2097357 (2005m:11089)

[5] B. Cho, S. Choi and C. H. Kim, Harmonic weak Maass-modular grids in higher level cases, Acta Arith. 160 (2013), no. 2, 129-141. MR3105331 
[6] SoYoung Choi and Chang Heon Kim, Congruences for Hecke eigenvalues in higher level cases, J. Number Theory 131 (2011), no. 11, 2023-2036, DOI 10.1016/j.jnt.2011.04.013. MR2825109(2012j:11101)

[7] SoYoung Choi and Chang Heon Kim, Basis for the space of weakly holomorphic modular forms in higher level cases, J. Number Theory 133 (2013), no. 4, 1300-1311, DOI 10.1016/j.jnt.2012.09.015. MR3004001

[8] P. Guerzhoy, Hecke operators for weakly holomorphic modular forms and supersingular congruences, Proc. Amer. Math. Soc. 136 (2008), no. 9, 3051-3059, DOI 10.1090/S0002-993908-09277-0. MR2407067(2009e:11089)

[9] Henryk Iwaniec, Topics in classical automorphic forms, Graduate Studies in Mathematics, vol. 17, American Mathematical Society, Providence, RI, 1997. MR 1474964 (98e:11051)

[10] Ken Ono, Unearthing the visions of a master: harmonic Maass forms and number theory, Current developments in mathematics, 2008, Int. Press, Somerville, MA, 2009, pp. 347-454. MR2555930(2010m:11060)

[11] Junichi Shigezumi, On the zeros of certain Poincaré series for $\Gamma_{0}^{*}(2)$ and $\Gamma_{0}^{*}(3)$, Osaka J. Math. 47 (2010), no. 2, 487-505. MR2722370 (2011k:11058)

[12] J. Shigezumi, On the zeros of certain modular functions for the normalizers of congruence subgroups of low levels, arXiv:0882.1307v2.

[13] W. Szpankowski, Mellin Transform and Its Applications, http://www.cs.purdue.edu/homes/ spa/papers/chap9.ps

Department of Mathematics Education, Dongguk University-Gyeongue, 123 Dongdaero, Gyeonguu, Gyeongbuk, 780-714, Republic of Korea

E-mail address: young@dongguk.ac.kr

Department of Mathematics and Research Institute for Natural Sciences, Hanyang University, Seoul 133-791, Republic of Korea

Current address: Department of Mathematics, Sungkyunkwan University, Suwon 440-746, Republic of Korea

E-mail address: chhkim@skku.edu 